\section{ANTERIOR SERRATUS PLANE BLOCK FOR AWAKE BREAST SURGERY; A CASE REPORT}

D Liotiri*. General Hospital of Rhodes, Rhodes, Greece

10.1136/rapm-2021-ESRA.15

Background and Aims We report a case of an 87-year-old female patient, ASA 3, with significant comorbidities who underwent awake right breast lumpectomy under anterior serratus plane block (ASPB), because of her high risk for general anaesthesia. Following a right total mastectomy 3 years ago, she presented with cancer recurrence; the lump was located close to the lateral aspect of previous mastectomy scar and close to axilla. The superficial ASPB was chosen because it blocks the nerves that innervate the lateral half of breast and the axilla. ${ }^{1}$

Methods Written informed consent was obtained by the patient for publication. The block was performed at the level of the fifth rib at the anterior axillary line with the patient supine. An ultrasound-guided in plane transverse approach was used. A total of $30 \mathrm{ml}$ ropivacaine $0.5 \%$ was administered in incremental boluses at the plane between the serratus anterior and the latissimus dorsi (figure 1). The patient was mildly sedated intraoperatively with $75 \mathrm{mcg}$ Fentanyl and $60 \mathrm{mcg}$ Clonidine and tolerated the procedure well.

Results Procedure and recovery were uneventful. She did not complain of any pain and she did not require any painkillers for the next 24 hours following the block. Her pain thereafter was controlled with rescue Paracetamol.

Conclusions Awake breast surgery under ASPB as single block technique is feasible when the surgery is limited to the lateral half of the breast and the axilla. Understanding the anatomy of the surgical technique is important to decide between the various regional techniques available for breast surgery.

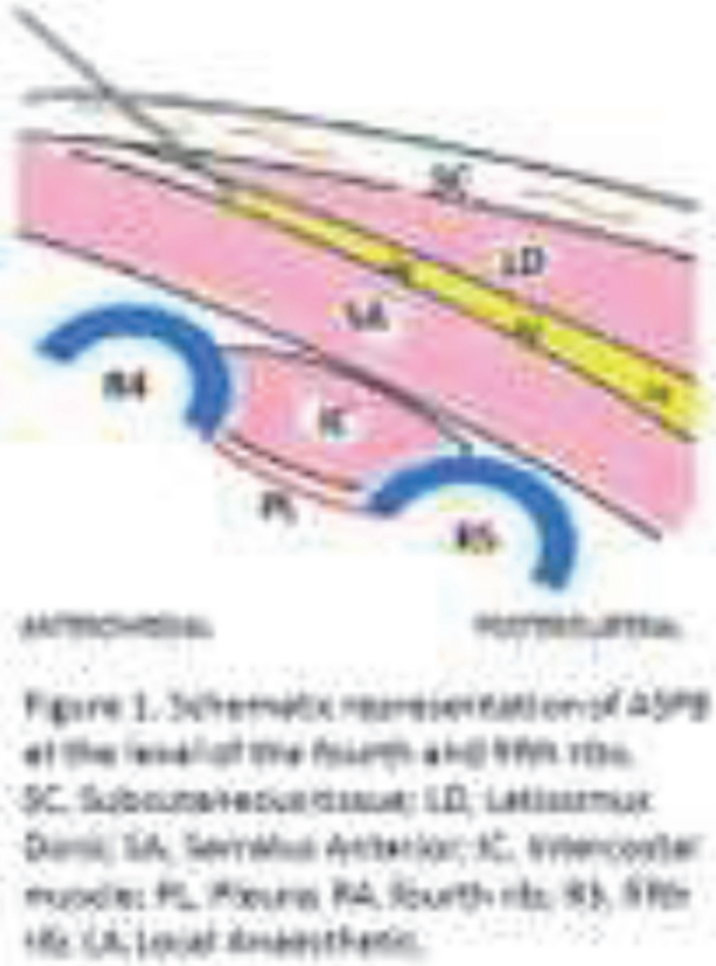

Abstract 15 Figure 1

\section{SUCCESSFUL REGIONAL ANESTHESIA MANAGEMENT FOR AWAKE SCAPULAR FRACTURE SURGERY: A CASE REPORT}

1,2,3,4E Yamak Altinuulluk, 1,5 D Nystad* ${ }^{*} 1,6$ LF Valdes-Vilchesa, ${ }^{1,7}$ F Gallucio, ${ }^{1,2,4,8}$ I Ince, ${ }^{1,9,10} \mathrm{~T}$ Ergonenc, ${ }^{1,11} \mathrm{CH}$ Salazar-Zamorano, ${ }^{1,12} \mathrm{M}$ Fajardo-Pérez. ${ }^{1}$ Morphological Madrid Research Center (MoMaRC), Madrid, Spain; ${ }^{2}$ Department of Outcomes Research, Cleveland Clinic, Cleveland, USA; ${ }^{3}$ Cerrahpasa Medical School, Istanbul, Turkey; ${ }^{4}$ Anesthesiology Clinical Research Office, Ataturk University, Erzurum, Turkey; ${ }^{5}$ Anesthesiology Department, University Hospital North Norway, Narvik, Norway; ${ }^{6}$ Anesthesiology Department, Hospital Costa del Sol, Agencia 54 Sanitaria Costa del Sol. Marbella, Malaga, Spain; ${ }^{7}$ Dipartimento di Medicina Sperimentale e Clinica: Firenze, 60 Università degli Studi di Firenze, toscana, Italy; ${ }^{8}$ Department of Anesthesiology and Reanimation, School of Medicine, Ataturk University, Erzurum, Turkey; ${ }^{9}$ Department of Anesthesiology, Akyazi Pain and Palliative Care Center, Sakarya, Turkey; ${ }^{10}$ Sakarya Training and Research Hospital, Sakarya, Turkey; ${ }^{11}$ Anesthesiology Department, 12 de Octubre Universitary Hospital, MADRID, Spain; ${ }^{12}$ Anesthesiology Department, Mostoles Universitary Hospital, Mostoles, Spain

\subsection{6/rapm-2021-ESRA. 16}

Background and Aims Since scapula innervation are very complex, the surgery for scapular fractures usually is done under general anesthesia. ${ }^{1}$ The aim was to perform successful regional anesthesia management with mild sedation for awake scapular fracture surgery with selective target block combinations.

Methods A 77-year-old female patient with a right scapula fracture was scheduled for an open reduction and internal fixation by posterior approach at the beach-chair position. With informed consent, we performed the upper trunk block, a selective supraclavicular, and T3 thoracic paravertebral block. Upper trunk block ${ }^{2}$ at the point where it bifurcates into the suprascapular nerve/posterior and anterior division was performed with the in-plane technique and $17 \mathrm{~mL}$ of Ropivacaine $0.5 \%$ in a semi-sitting position in the supraclavicular fossa. (figure 1A). In this case, the supraclavicular nerve, divided

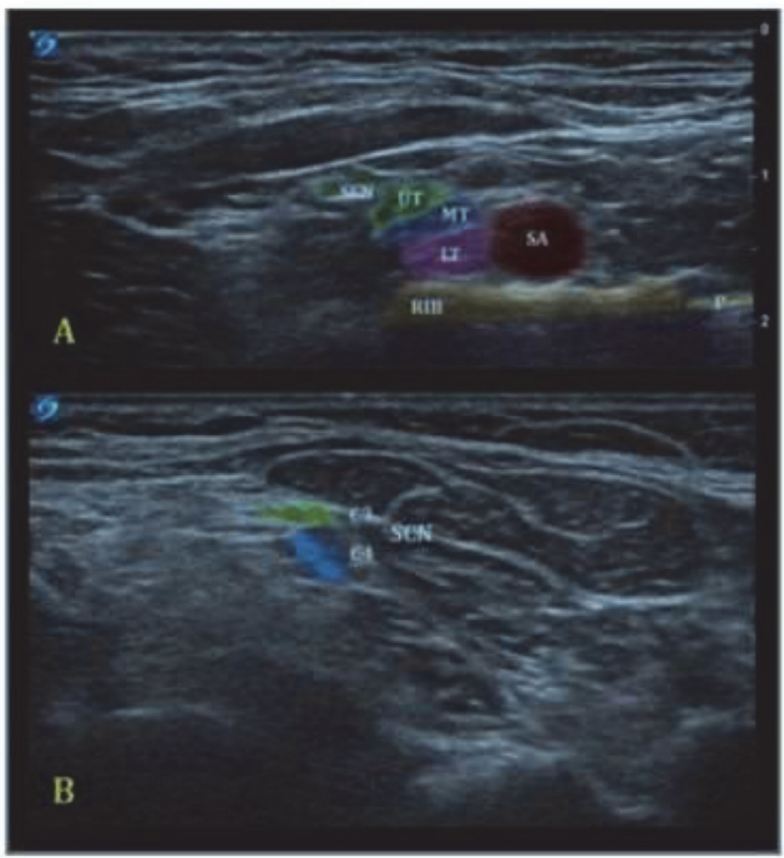

Abstract 16 Figure 1 A. Ultrasound image of Brachial Plexus, $\mathrm{SN}_{i}$ suprascapular nerve, UTa; Upper trunk anterior division UTp; Upper trunk posterior division MT; medial trunk LT; Lateral trunk SA; Subclavian Artery B. Ultrasound image of two branches (from C3 and C4) of supraclavicular nerve, SCN; Supraclavicular nerve 
into two branches, was blocked separately by $3 \mathrm{~mL}$ Ropivacaine $0.5 \%$. (figure $1 \mathrm{~B}$ ) The thoracic paravertebral block at T3 with a total of $20 \mathrm{~mL}$ Ropivacaine $0.5 \%$ was performed at the sitting position by in the oblique sagittal in-plain technique from lateral to medial.

Results The patient was completely awake (Richmond agitation scale 0) during the surgery and no pain in the postoperative care unit. (NRS 0/10). The acetaminophen $1 \mathrm{~g}$ orally just once given after almost 5 hours of surgery and she was discharged home 24 hours after the surgery.

Conclusions The selective target block combinations might be considered for all structures with complex innervation, such as scapula.

\section{ULTRASOUND GUIDED ASSOCIATED TO THE FLUOROSCOPY COULD IMPROVE THE EFFICACY AND SAFETY OF THE RADIOFREQUENCY APPLICATION ON THE GANGLION IMPAR: A CASE REPORT}

${ }^{1} \mathrm{P}$ Fusco, ${ }^{2} \mathrm{~F}$ Angelucci*, ${ }^{2} \mathrm{~S}$ Tullj, ${ }^{3} \mathrm{~W}$ Ciaschi, ${ }^{2} \mathrm{~F}$ Marinangeli. ${ }^{1} 1$ Department of Anesthesia and Intensive Care, San Salvatore Academic Hospital, L'Aquila, Italy; ${ }^{2}$ University of L'Aquila, Department of Life, Health and Environmental Sciences, L'Aquila, Italy; ${ }^{3}$ Department of Anesthesia and Intensive Care, F. Spaziani Academic Hospital, Frosinone, Italy

\subsection{6/rapm-2021-ESRA.17}

Background and Aims Chronic perineal pains is a painful condition. In this study, we aimed to assess the effect the ultrasound associated to the fluoroscopy for improve the effectiveness and the safety of the radiofrequency on the ganglion impar in the patient with perianal pain ${ }^{1}$, who did not respond to standard drug therapies ${ }^{2}$

Methods The case was a 79-year-old woman with history of continuous stabbing pain in the anal region. At her first visit, the VAS was $9 / 10$.

The patient was in the prone position on the operating table.

After disinfection in the region of the sacral, a sterile echographic linear probe was placed transversely on the lower portion of the sacred until the identification of the sacral cornets.

Then, the probe is rotated in a longitudinal position until the identification of the sacrococcygea joint.Under constant ultrasound guidance and fluoroscopy, a 22 G $100 \mathrm{~mm}$ radiofrequency needle was introduced in the precoccigeo space near the ganglion impar.

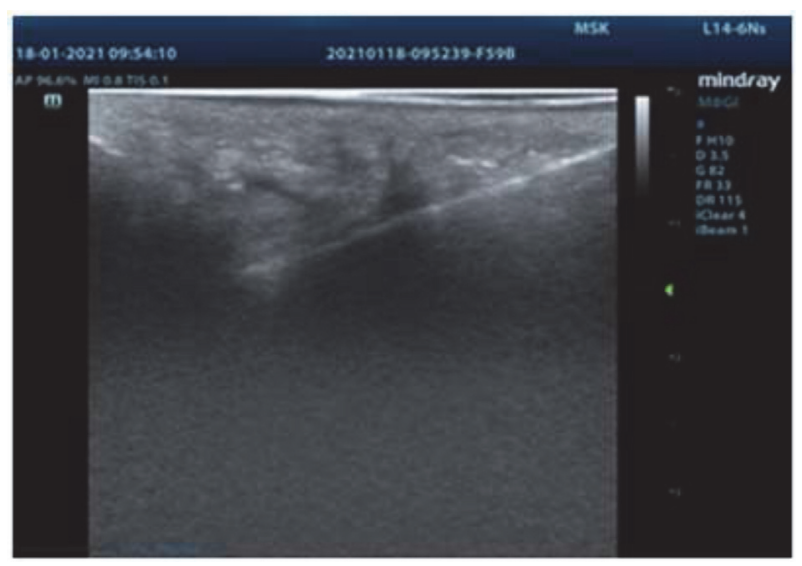

Abstract 17 Figure 1

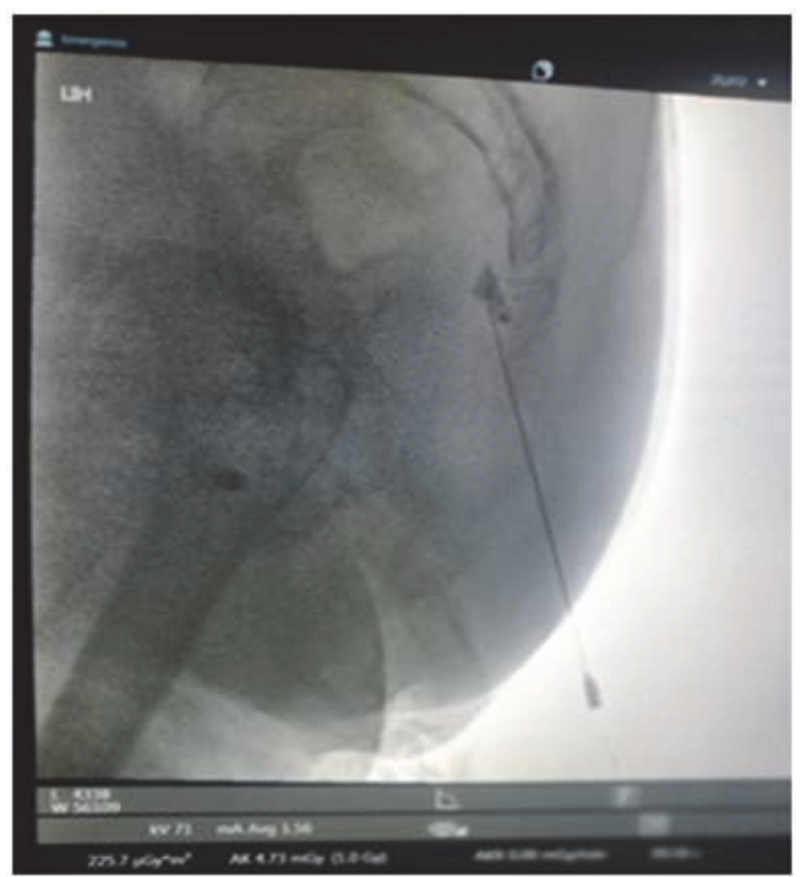

Abstract 17 Figure 2

The position of the needle tip was confirmed by injecting $1 \mathrm{~mL}$ of radiopaque dye in the retroperitoneal space.Then, a thermocouple connected to a radiofrequency generator was inserted to reproduce the patient's paresthesia via sensitive stimulation, $0.4-0.5 \mathrm{mV}$. The radiofrequency was performed at $90^{\circ} \mathrm{C}$ for 90 seconds.

Results At the end of the procedure the patient reported VAS 0 .

Conclusions The result suggests that ultrasound guided combined with fluoroscopy enhances the safety and accuracy of radiofrequency on the ganglion impar. Furthermore, it significantly reduces radiation exposure for both patients and operator.

\section{THE EFFICACY OF THE ULTRASOUND-GUIDED INTERMEDIATE CERVICAL PLEXUS BLOCK FOR CAROTID ENDARTERECTOMY}

D Mathew*, MH Wong. Khoo Teck Puat Hospital, Singapore, Singapore

\subsection{6/rapm-2021-ESRA.18}

Background and Aims Carotid Endarterectomy (CEA) is a prophylactic surgery performed to prevent embolic stroke in patients with atheromatous disease in the carotid bifurcation. Reducing perioperative stress is critical in minimizing cardiovascular and cerebrovascular complications. Both superficial and deep cervical plexus block (CPB) have been described to provide effective analgesia and anaesthesia for head and neck surgery. Recently, an intermediate cervical plexus block has been described as a variant. We report a case that was successfully managed with ultrasound guided intermediate cervical plexus block for CEA in a high-risk patient.

Methods Patient consent was obtained for the case report. A 63-year-old male patient was scheduled for a left sided CEA with synthetic patch grafting. The patient presented with a history of right sided sensory-motor syndrome with a CT four 